\title{
Commentary
}

\section{Comments on 'Relevance of Context-Bound Loci to Topical Potential in the Argumentation Stage'}

\author{
C. ANDONE \\ Department of Speech Communication, Argumentation Theory and Rhetoric, \\ University of Amsterdam, Amsterdam, The Netherlands \\ E-mail: C.Andone@uva.nl
}

The paper written by Eddo Rigotti stands as a skillfully contrived and original piece of work, a valuable part of a larger project concerned with the systematic construction of a course dealing with argumentation in different fields of application. One focus of the project is to develop a model of topics envisaged as an argument generator that could assist the arguer, as the author claims in the definition provided, "to identify all (theoretically possible) relevant arguments in favor and against any standpoint", being "generated by specifying their inferential structure through a system of loci". In this article, emphasis is given to the topical potential of strategic maneuvering in the context of the argumentation stage of a discussion by establishing a relationship between argument choice and the communicative situation within which the argumentative intervention takes place.

To be more precise, Rigotti's aim is to deal with five main aspects: (a) the relationship between strategic maneuvering and his new version of topics by bringing into the foreground the connection between topics and the communicative context, (b) the clarification of the key concepts from the theoretical framework, (c) the presentation of a taxonomy of loci, (d) an analytical approach to topics in the argumentation stage and (e) the clarification of the use of the argument generator. In my commentary I first discuss the following three points: the complexity of the model of topics that is proposed, the view of the communicative context that is put forward and the classification of loci based on semantic and pragmatic aspects. Finally, I react to the analysis of the example given.

My comment concerning the complexity of the model in connection with which an outline is given, is related to the motivation and distinctive features of its design. It is fully true, as Rigotti implies, that the study of topics in the ancient and medieval tradition received a comprehensive, but very complex approach, which calls for a more consistent and substantial model that would incorporate elements from modern semantics and pragmatics. The aim of the model presented in this paper to consider new argumentative practices in the communicative context where the argumentative discussion takes place is indeed 
achieved in the proposal. However, I think it needs to be more precisely shown how the model offers a simplification of the process of invention and how it facilitates this process, which stands as the main purpose of the taxonomy of loci presented. It looks rather as if the proposed model, consistent and coherent in itself, gives a more intricate account of the inventional process than it is claimed, which makes it more complicated than previous proposals.

My second comment concerns the communicative context. What I would like to question here is the relevance for the model of topics. According to Rigotti, this context is constituted of two dimensions, the institutional dimension and the interpersonal dimension. With reference to the institutional dimension, the author explains that "the interaction field operates through a series of interaction schemes which aim at the fulfillment of the shared goals". In the way the communicative context is represented, though, the interaction schemes and the interaction field are portrayed as two sub-components of the institutional dimension. Moreover, the connection shown graphically between the implementing subjects related to the interpersonal dimension and the institutional dimension remains unexplained, as does the agency relationship that they maintain with the interaction field.

I totally concede to the idea put forward by Rigotti that the production of arguments supporting the standpoint in connection with a particular dimension of the context is of particular relevance in designing arguments in the argumentation stage. While it is claimed that "all components of the context are relevant in designing arguments in the argumentation stage", it is not made clear, however, in the analyses of the two examples provided, how the two dimensions are exploited and influence one other, and if that is indeed the case, how they could be both integrated into a complex syllogism of the kind presented. Moreover, it remains to be explained how the shared goals are determined and how can one tell what the main goal is and what the subordinate goal is.

My third comment concerns Rigotti's classification of loci. As part of the model, a taxonomy of loci as it appears in the Medieval literature is presented, comprising extrinsic, intrinsic and mixed loci. For each of the three categories, a new term is proposed, offering a division into syntagmatic, paradigmatic and complex loci. If, as indicated in the classification, loci are distinguished according to their proximity to the standpoint, one would like to know how the three modern terms correspond to the old labels. Besides, Rigotti claims that loci are built on the basis of the different hooking points that link the argument to the standpoint, but, to give just one example, it is not easy to see the dependence of the argument on the hooking point of the standpoint in the case of the paradigmatic locus from metaphor, 
unlike in the case of the complex locus of authority in which the authority is the hooking point and the argument relies on the authority of the arguer.

I now turn to the analysis of the example as proposed by Rigotti in this paper. The graphical representation that highlights the different components and the arguments that support the unexpressed standpoint shows that 'the minor premise A' and 'the minor premise B' are generated by the endoxon, although it is claimed that a conjunction relation holds. The coordinative relation suggested to hold is not represented either and the question remains what the relationship between the two premises is. The same holds for the minor premise shown which is claimed to be derived from the maxim. An issue arises in connection with its content. It is explained that 'first conclusion $\mathrm{A}^{\text {' }}$ and 'first conclusion B' together form a complex minor premise, but if that is the case, it can only be the final conclusion, which makes the insertion of an intermediary minor premise redundant. Besides these aspects, if an endoxon is that which is already in the shared opinion and has the form of a general claim as shown in the content of endoxon $\mathrm{A}$, endoxon $\mathrm{B}$ does not function as such. One can also imagine that 'first conclusion B' could be 'Ticino decided to create a university in the same way that a young couple wants to have a baby'. Moreover, only if 'first conclusion A' specifying that 'adult children are expected not to discuss the decision of having a baby with their parents' is replaced by 'it is natural for adult children not to discuss the matter of having a baby with their parents', the 'final conclusion' can hold. Otherwise, it does not follow from the combination of the major premise with the minor premise that 'Ticino's behavior is natural'.

I believe that Eddo Rigotti has written a rich paper, the scope of which was to provide a comprehensive model of topics driven by the importance underlined at the beginning of the paper of the topical potential in the argumentation stage. As becomes clear from the paper, two levels are considered in the construction of an argumentative strategy: an inferential level and a rhetorical level. In my opinion, it can be made more prominent that the syllogism deriving from the endoxon gives the argument its persuasive power by making more prominent the integration with the syllogism deriving from the maxim. If that could be achieved, the role of strategic maneuvering would also be made more prominent. It remains to be concluded, though, how the two levels can be connected in such a way that the advantages of the proposed model are emphasized. 